\title{
OPEN Mass production-enabled computational spectrometers based on multilayer thin films
}

\begin{abstract}
Cheolsun $\mathrm{Kim}^{1}$, Pavel $\mathrm{Ni}^{1}$, Kang Ryeol Lee ${ }^{2}$ \& Heung-No Lee ${ }^{1 \bowtie}$
Multilayer thin film (MTF) filter arrays for computational spectroscopy are fabricated using stencil lithography. The MTF filter array is a $6 \times 6$ square grid, and 169 identical arrays are fabricated on a single wafer. A computational spectrometer is formed by attaching the MTF filter array on a complementary metal-oxide-semiconductor (CMOS) image sensor. With a single exposure, 36 unique intensities of incident light are collected. The spectrum of the incident light is recovered using collected intensities and numerical optimization techniques. Varied light sources in the wavelength range of 500 to $849 \mathrm{~nm}$ are recovered with a spacing of $1 \mathrm{~nm}$. The reconstructed spectra are a good match with the reference spectra, measured by a grating-based spectrometer. We also demonstrate computational pinhole spectral imaging using the MTF filter array. Adapting a spectral scanning method, we collect 36 monochromatic filtered images and reconstructed 350 monochromatic images in the wavelength range of 500 to $849 \mathrm{~nm}$, with a spacing of $1 \mathrm{~nm}$. These computational spectrometers could be useful for various applications that require compact size, high resolution, and wide working range.
\end{abstract}

Spectrometers are powerful tools for remote sensing and medical applications ${ }^{1-3}$. However, these uses are restricted to research and development due to limitations based on the spectrometers' bulky size, high cost, and long measuring time. There have been tremendous efforts to overcome spectrometer limitations and go beyond restricted applications ${ }^{4-21}$. One promising candidate to achieve this is optical filter array-based spectrometers: complementary metal-oxide-semiconductor (CMOS) image sensors with a filter array used as a spectrometer. These spectrometers are smaller and have faster measuring times, so they are useful in portable applications, such as on-site detection and small unmanned aerial vehicle (UAV)-based remote sensing. However, the number of filters that can be attached to a CMOS image sensor is limited due to its small sensing area. Thus, these spectrometers offer a low spectral resolution.

Over the past decade, computational approaches ${ }^{22,23}$ have been adapted for filter-based spectrometers. The spectral resolution in conventional filter array-based spectrometers has been improved using computational approaches. New optical filter types have been proposed that work well in computational approaches and achieve further improvements ${ }^{6-8,10,11,13,14,16,21}$. Unlike conventional optical filters, which selectively transmit incident light in specific wavelengths and reflect the remaining wavelengths, these filters, called random spectral filters, modulate and transmit incident light with unique spectral features in the entire wavelength ranges of interest. Various types of random spectral filters have been proposed, such as etalon filters ${ }^{10,11,20}$, quantum dot filters ${ }^{8,21}$, photonic crystal slabs ${ }^{7,9,14,16}$, and multilayer thin films (MTF) filters ${ }^{6,13}$. The spectral resolvability of computational spectroscopy has been successfully demonstrated using random spectral filters with low correlation among filters.

In contrast to transmission functions of an etalon filter, which consists of repetitive narrow peaks, and a quantum dot filter, which consists of a broadband peak, the fabricated random spectral filter has a transmission function of multiple peaks with various full widths at half maximums (FWHMs) and has a large difference between maximal and minimal transmission in the transmission function. By utilizing the computational approaches, a wide wavelength range can be covered with a small number of MTF filters. In this work, a small number of MTF-based random spectral filters were fabricated in the form of an array. 169 identical filter arrays, consisting of 36 MTF filters, were fabricated on a single wafer. We realized MTF filters by stacking multiple layers of two alternating materials with high and low refractive indices. Using stencil lithography based on shadow masks, we could fabricate MTF filters with different spectral features simultaneously as a filter array form. This idea of random spectral filters can be applied to various wavelength ranges by changing the MTFs' design properties.

${ }^{1}$ School of Electrical Engineering and Computer Science, Gwangju Institute of Science and Technology, Gwangju 61005, South Korea. ${ }^{2}$ SNI Co., Ltd., Seongnam, Gyeonggi 13509, South Korea. ${ }^{\circledR}$ email: heungno@ gist.ac.kr 
a

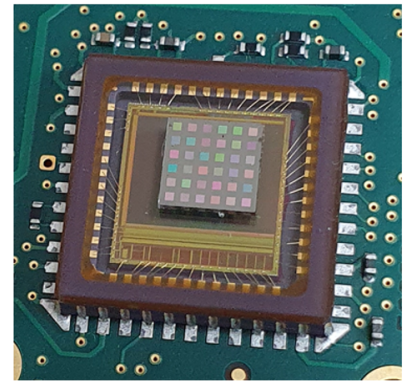

b

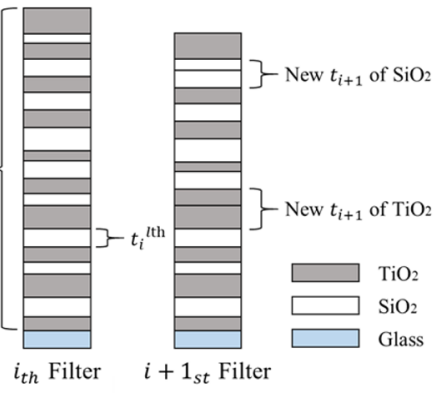

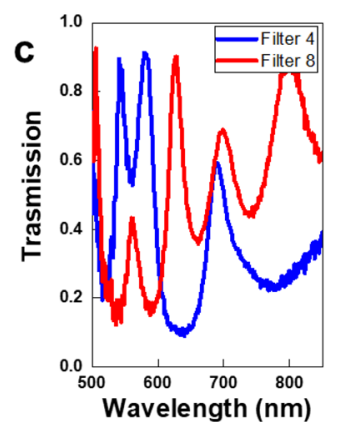
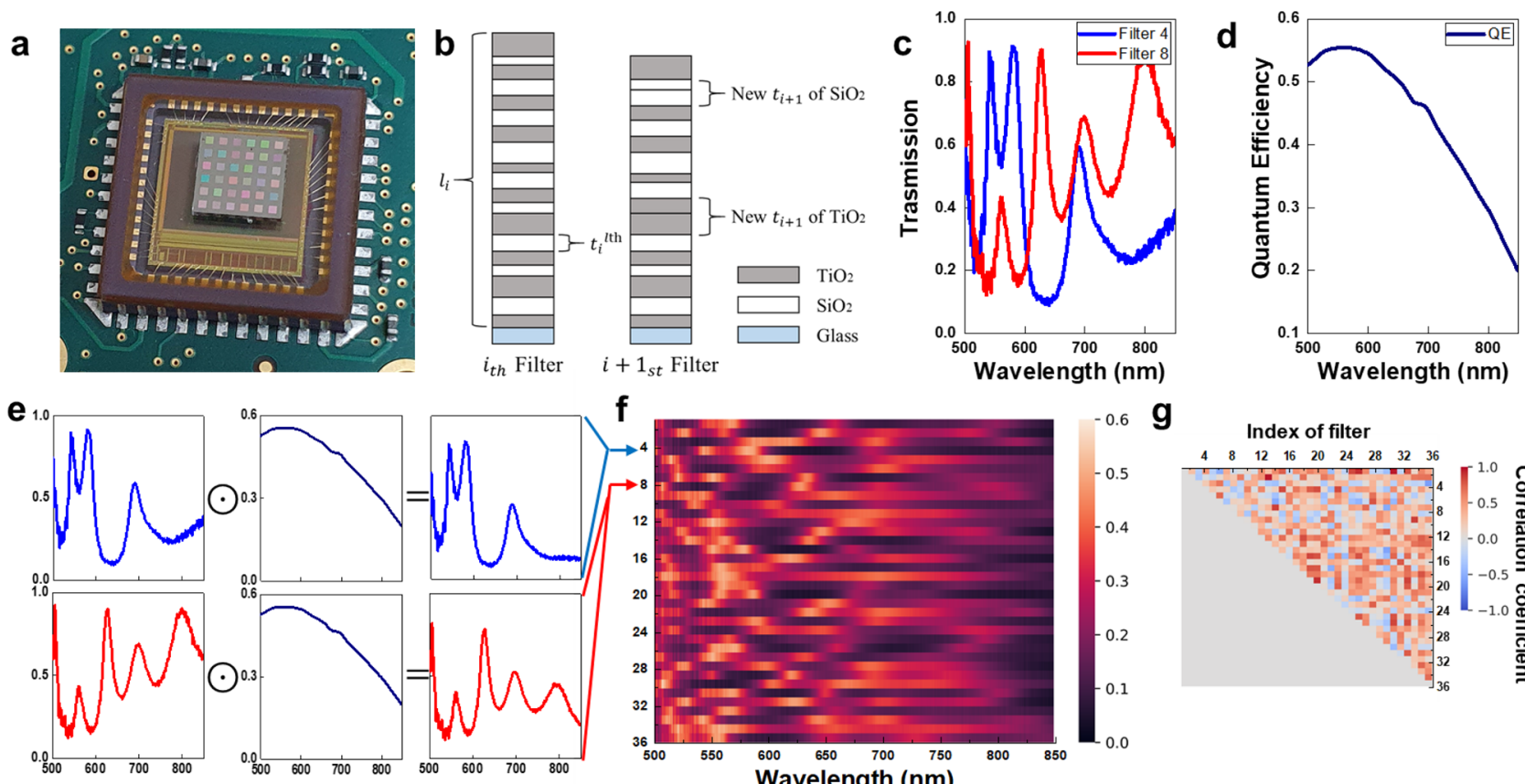

f
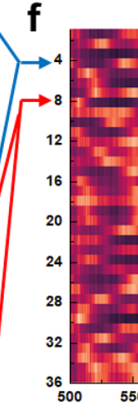

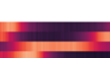

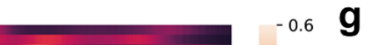

g

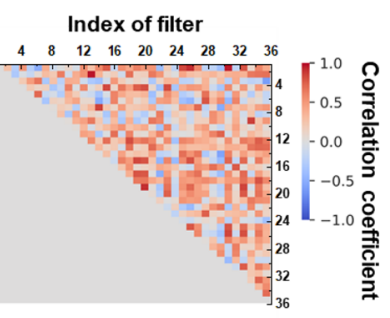

Figure 1. Multilayer thin films (MTF) based computational spectrometer. (a) Photograph of the MTF filter array, which is directly attached to the CMOS image sensor. (b) Schematic of MTF filters: $\mathrm{TiO}_{2}$ and $\mathrm{SiO}_{2}$ are deposited on a glass substrate with design properties such as the number of layers of the $i$-th MTF filter $\left(l_{i}\right)$ and thickness of the $l$-th layer of the $i$-th MTF filter $\left(t_{i}^{l \text { th }}\right)$. (c) Two transmission functions of MTF filters. (d) Spectral response of the CMOS image sensor. (e) Spectral sensitivity of an MTF filter with the CMOS image sensor, which can be calibrated by element-wise multiplication of the transmission function and the spectral response of the CMOS image sensor. (f) Heatmap of the sensing matrix. Each row represents the spectral sensitivity with respect to wavelength. (g) Upper triangular matrix of correlation coefficients which are pairwise compared among rows of the sensing matrix.

According to the usage of MTF filters, they can also be mass-produced in various shapes using stencil lithography techniques.

Here, we demonstrate the competence of spectral reconstructions over the wavelength range of 500 to 849 $\mathrm{nm}$ using a fabricated filter array. The fabricated filter array consists of 36 MTF filters in the shape of a square. Varied spectra of incident light such as monochromatic, broadband, and continuous light is used to test the filter array's reconstruction performances. Additionally, we perform pinhole spectral imaging using the filter array, showing that computational spectral imaging is possible.

\section{Results}

Spectrometers based on MTF filters. The computational spectrometer consists of MTF filters and the CMOS image camera. As shown in Fig. 1a, MTF filters are in the form of an array and are directly attached to the CMOS image sensor. Each filter has unique spectral features that can be realized by stacking multiple layers of thin films. A schematic of the MTF filter is shown in Fig. 1b. The transmission function of the $i$-th MTF filter is determined by design properties, such as the number of layers $\left(l_{i}\right)$ and the thickness of the $l$-th layer $\left(t_{i}^{l \text { th }}\right)$. Using the transfer-matrix method, the transmission function of the MTF filter can be calculated ${ }^{24,25}$. We choose a set of different design properties to produce a set of filters with a unique transmission function. Let us denote the transmission function of the $i$-th MTF filter in the wavelength range $\lambda=\left[\lambda_{1}, \lambda_{2}, \ldots, \lambda_{N}\right]$ as $\mathbf{T}_{i}=\left[T_{i}\left(\lambda_{1}\right), T_{i}\left(\lambda_{2}\right), \ldots, T_{i}\left(\lambda_{N}\right)\right]$. Figure 1c shows two measured transmission functions of the MTF filters (see Sect. 4 for measuring transmission functions of MTF filters). The intensity, $y_{i}$, measured by CMOS image sensor for an unknown incident spectrum $\mathbf{x}=\left[x\left(\lambda_{1}\right), x\left(\lambda_{2}\right), \cdots, x\left(\lambda_{N}\right)\right]^{T}$, can be expressed as:

$$
y_{i}=\sum_{k=1}^{N} T_{i}\left(\lambda_{k}\right) Q\left(\lambda_{k}\right) x\left(\lambda_{k}\right),
$$

where $\mathbf{Q}=\left[Q\left(\lambda_{1}\right), Q\left(\lambda_{2}\right), \ldots, Q\left(\lambda_{N}\right)\right]$ is the spectral response of CMOS image sensor in the wavelength range $\lambda$. The spectral response is represented in Fig. 1d. Let us set $R_{i}\left(\lambda_{k}\right)=T_{i}\left(\lambda_{k}\right) Q\left(\lambda_{k}\right)$, where $R_{i}\left(\lambda_{k}\right)$ represents spectral sensitivity of the $i$-th filter of the CMOS image sensor at the wavelength $\lambda_{k}$, the Eq. (1) becomes $y_{i}=\sum_{k=1}^{N} R_{i}\left(\lambda_{k}\right) Q\left(\lambda_{k}\right)$. Considering an $M$ number of filters, there is a set of $M$ equations for $i=1,2, \ldots, M$. The set of $M$ equations can be represented in matrix formation: 

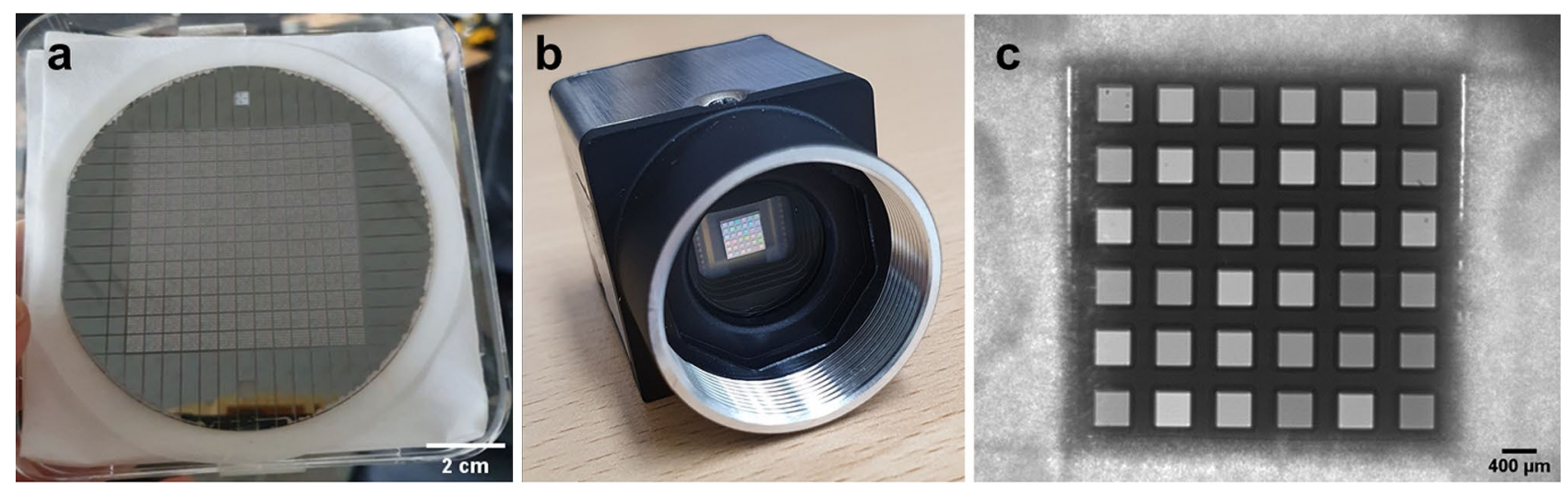

Figure 2. Fabricated MTF filter array. (a) 169 identical MTF filter arrays fabricated in a single wafer. (b) Photograph of the CMOS image camera with the fabricated MTF filter array. (c) Monochrome image of the fabricated MTF filter array illuminated by a halogen light source.

$$
\left[\begin{array}{c}
y_{1} \\
\vdots \\
y_{M}
\end{array}\right]=\left[\begin{array}{ccc}
R_{1}\left(\lambda_{1}\right) & \cdots & R_{1}\left(\lambda_{N}\right) \\
\vdots & \ddots & \vdots \\
R_{M}\left(\lambda_{1}\right) & \cdots & R_{M}\left(\lambda_{N}\right)
\end{array}\right]\left[\begin{array}{c}
x\left(\lambda_{1}\right) \\
\vdots \\
x\left(\lambda_{N}\right)
\end{array}\right],
$$

where $\mathbf{y} \in \mathbb{R}^{M \times 1}$ is a column vector with measured intensities from $M$ filters and $\mathbf{R} \in \mathbb{R}^{M \times N}$ is the sensing matrix where each row represents the spectral sensitivity with respect to the wavelength. The spectral sensitivity can be calibrated by element-wise multiplication of the transmission functions of MTF filters and the spectral response of the CMOS image sensor, as depicted in Fig. 1e,f.

Conventional spectrometers read out $\mathbf{y}$ as the incident spectrum $\mathbf{x}$. In order to make the measured intensities $\mathbf{y}$ as close as possible to the incident spectrum $\mathbf{x}$, the sensing matrix $\mathbf{R}$ should be an identity matrix with the dimension of $N \times N(M=N)$. This means that $N$ number of filters are needed in conventional manners. In practice, it may be difficult to fabricate a narrow FWHM filter and, since the number of filters required increases as the wavelength of interest increases, it is more challenging to make a compact spectrometer operating in a wide wavelength range. Unlike conventional filter-based spectrometers, computational spectrometers modulate and measure a wide wavelength range of the incident spectrum using a small number of MTF filters. We consider the sensing matrix $\mathbf{R}$ with dimensions $M \times N(M<N)$. The set of $M$ equations becomes an underdetermined problem. Reconstruction algorithms $\mathrm{s}^{26-28}$ can be applied to restore the incident spectrum in high resolution by solving the underdetermined problem.

Figure if shows the heatmap of the sensing matrix of the fabricated MTF filter array-based computational spectrometer. Each row represents the spectral sensitivity with respect to wavelength. The correlation coefficients for each pair of two rows of the sensing matrix are shown as the upper triangular matrix in Fig. 1g. The average value of the correlation coefficients is 0.231 , which can be described as a weak or moderate correlation among sensitivities. With the weakly correlated spectral sensitivities, the incident spectrum was measured as unique intensities, which allow the reconstruction algorithms to work effectively.

Fabrication of MTF filter arrays. We fabricated 169 identical filter arrays on a single wafer, as shown in Fig. 2a. The filter array is the shape of a $6 \times 6$ square grid. The size of the square is $400 \times 400 \mu \mathrm{m}^{2}$, and the space between the squares is $300 \mu \mathrm{m}$. Accordingly, the size of filter array is $4.5 \times 4.5 \mathrm{~mm}^{2}$. To fabricate filter arrays, we use $\mathrm{TiO}_{2}$ and $\mathrm{SiO}_{2}$ as a high and low refractive index materials, respectively. The refractive indices for $\mathrm{TiO}_{2}$ and $\mathrm{SiO}_{2}$ are approximately 2.6 and 1.45 at $600 \mathrm{~nm}$, respectively.

Unlike etalon filters that were fabricated by changing the thickness of interspacing dielectric layers ${ }^{20,29}$, we fabricated the MTF filters by changing the number of layers and thicknesses of layers. An MTF filter with a unique transmission function can be obtained by repeatedly alternating the two materials and depositing them with different thicknesses. 36 MTF filters with unique transmission functions were fabricated by selectively omitting certain layers of different MTF filters using shadow masks during the deposition of the MTF filter array. As shown in Fig. 1b, the upper and lower layers sum up to form one layer with a different thickness by omitting an intermediate layer. The designed thicknesses of layers for MTF filters are shown in Supplementary Information Table $\mathrm{S} 1$. The deposition process for creating filter arrays follows.

$\mathrm{TiO}_{2}$ and $\mathrm{SiO}_{2}$ films were deposited onto a borosilicate glass wafer whose refractive index is approximately 1.472 at $588 \mathrm{~nm}$. In order to distinguish where the material should be deposited, shadow masks were used. The desired thickness of $\mathrm{TiO}_{2}$ is deposited on the desired locations using direct current (DC) magnetron sputter. For $\mathrm{TiO}_{2}$ deposition, a Ti target was sputtered in a mixture of argon ( $\left.\mathrm{Ar}\right)$ and oxygen $\left(\mathrm{O}_{2}\right)$. The mixture gas flow of $188 \mathrm{sccm}$ of $\mathrm{Ar}$ and $12 \mathrm{sccm}$ of $\mathrm{O}_{2}$ was utilized and the DC power was $700 \mathrm{~W}$. The $\mathrm{TiO}_{2}$ deposition is performed only on the desired region designated by the shadow mask. Then, the shadow mask is changed, with different patterns on the other mask, and we deposit $\mathrm{SiO}_{2}$ at the intended thickness. Radio frequency (RF) magnetron sputter was used for the $\mathrm{SiO}_{2}$ deposition. A Si target was sputtered in a mixture of $\mathrm{Ar}$ and $\mathrm{O}_{2}$. The mixture gas 
flow of $185 \mathrm{sccm}$ of Ar and $15 \mathrm{sccm}$ of $\mathrm{O}_{2}$ was utilized. The RF power was $300 \mathrm{~W}$. The deposition is repeated 17 additional times by changing the shadow mask and alternating between $\mathrm{TiO}_{2}$ and $\mathrm{SiO}_{2}$. Hence, we conducted ten individual depositions of $\mathrm{TiO}_{2}$ and nine individual depositions of $\mathrm{SiO}_{2}$. The number of shadow masks used in depositions was 19. After completing thin film deposition, we coated the surface of thin films with a photoresist. Germanium $(\mathrm{Ge})$ was deposited over the entire wafer area using an e-beam evaporator. Lift-off of the photoresist was performed by soaking the deposited wafer in acetone. When the photoresist was washed away, Ge deposited on the top of the photoresist was lifted off and washed. After lift-off, a square grid of Ge with the size of $400 \mu \mathrm{m}$ and spacing of $300 \mu \mathrm{m}$ was formed. The Ge grid was formed to separate MTF filters and prevent incident lights from entering among MTF filters. The wafer cleaning process was then performed, and, finally, the wafer was diced to produce MTF filter arrays.

Unlike the previous work in that SiNx was used as the high refractive index material to fabricate an MTF filter array ${ }^{13}$, we used $\mathrm{TiO}_{2}$ as the high refractive index material and could reduce the number of layers for realizing the unique transmission functions. In addition, using stencil lithography, MTF filter arrays could be fabricated in a simplified process that does not involve an etching process.

The MTF filter array-based spectrometer was built by attaching the fabricated MTF filter array to the front of a CMOS monochrome camera, as shown in Fig. 2b. Figure $2 \mathrm{c}$ is a monochrome image of the fabricated MTF filter array illuminated by a halogen light source. The image was taken by the CMOS monochrome camera, whose number of pixels is $1280 \times 1024$. As shown in Fig. 2c, we measure uniform intensity using pixels under a single MTF filter. Also, the pixels have unique intensity according to the MTF filter. Using these unique intensities from MTF filters, we can reconstruct the spectrum of unknown incident light.

Spectral reconstruction experiments. Here, we address the spectral resolvability of the MTF filter array-based computational spectrometer. An unknown spectrum, $\mathbf{x}$, consisting of $350(N=350)$ spectral components from wavelengths ranging 500 to $849 \mathrm{~nm}$, is retrieved using measured intensities y with size $36(M=36)$. For retrieving the unknown spectrum, we use a sparse representation-based $l_{1}$-norm minimization problem. $\mathbf{x}$ can be represented as the multiplication of a sparsifying basis $\mathbf{G} \in \mathbb{R}^{N \times N}$ and a sparse signal $\mathbf{s} \in \mathbb{R}^{N \times 1}$, i.e., $\mathbf{x}=$ Gs. Then, Eq. (1) becomes $\mathbf{y}=\mathbf{R G s}$. The solution of the sparse signal, $\hat{\mathbf{s}}$, can be retrieved by solving the following minimization problem with nonnegativity constraints:

$$
\min _{\mathbf{s}}\|\mathbf{y}-\mathbf{R G s}\|_{2}^{2}+\gamma\|\mathbf{s}\|_{1} \text { subject to } s_{k} \geq 0 \text { for } k=1,2, \ldots, N,
$$

where $\gamma$ is the non-negative regularization parameter and $\|\mathbf{s}\|_{p}$ is defined as $\left(\sum_{k=1}^{N}\left|s_{k}\right|^{p}\right)^{1 / p}$. We use the collection of Gaussian distribution functions for the sparsifying basis $\mathbf{G}^{4}$. The linear combination of Gaussian distribution functions represents the line shape of the spectrum. The retrieved spectrum $\hat{\mathbf{x}}$ is G $\hat{\mathbf{s}}$. There exist open-source program that can be easily accessed to solve the numerical optimization problem ${ }^{30,31}$. In this work, we use the 11_ls package ${ }^{31}$ to solve the problem. All the spectral reconstructions were done in MATLAB R2017b with an Intel Core i7-5820 K CPU computer. The reconstruction of a single spectrum was done within $\sim 0.1 \mathrm{~s}$.

Before conducting spectral reconstructions, we first measured the transmission functions of MTF filters. A beam from the halogen light source (KLS-150H-LS-150D, Kwangwoo) was fed into a monochromator (MMAC200, Mi Optics). From the monochromator, a monochromatic light with an FWHM of $4 \mathrm{~nm}$ was generated. After passing through a collimator, the collimated monochromatic light was fed into the CMOS monochrome camera (EO-1312M, Edmund optics). Using the CMOS camera, we measured the light intensities with and without the MTF filter array. Then, the transmission $T$ of $i$-th filter at wavelength $\lambda_{k}$ is calculated by:

$$
T_{i}\left(\lambda_{k}\right)=\frac{I W F_{i}\left(\lambda_{k}\right)-B I_{i}\left(\lambda_{k}\right)}{I W O F_{i}\left(\lambda_{k}\right)-B I_{i}\left(\lambda_{k}\right)},
$$

where $I W F_{i}, I W O F_{i}$, and $B I_{i}$ are intensity with $i$-th filter, intensity without $i$-th filter, and background intensity, respectively. Using the monochromator, we could generate series of monochromatic light at the peak locations from 500 to $849 \mathrm{~nm}$ with the step of $1 \mathrm{~nm}$. We captured 350 pairs of monochrome images with and without filters in the wavelength range of 500 to $849 \mathrm{~nm}$. Using Eq. (4), we could obtain transmission functions of 36 MTF filters. The transmission functions were calibrated by element-wise multiplication of the spectral response of the CMOS image sensor as shown in Fig. 1e.

To analyze the dual-peak resolution of the fabricated MTF filter array spectrometer, we conducted simulations of dual-peak spectra reconstructions. Figure 3a shows an example of a dual-peak spectrum. The recovery performances were investigated for noisy environments ranging from 10 to $35 \mathrm{~dB}$ of signal-to-noise ratios (SNRs), more details are in the Methods section. The root mean squared error (RMSE), which is defined as $\sqrt{\left\|\mathbf{x}_{\text {refer }}-\mathbf{x}_{\text {recon }}\right\|_{2}^{2} / N}$, was used to evaluate the performances. The result of dual-peak spectra reconstructions with respect to SNRs is shown in Fig. 3b. We considered four kinds of dual-peak spectra. The FWHMs of a peak were 1 and $2 \mathrm{~nm}$, respectively and the gaps between peaks were 2 and $3 \mathrm{~nm}$, respectively. For each kind of dualpeak, spectra were created by changing the location of dual-peak in the wavelength range of 500 to $849 \mathrm{~nm}$. The reconstructions were performed on all these spectra, and RMSEs were calculated. We averaged the RMSEs and regarded the average RMSE value as the performance of the fabricated MTF filter array to reconstruct dual-peak in noisy environments. As shown in Fig. 3b, the average RMSEs of dual-peak with the FWHM of $1 \mathrm{~nm}$ and the gap of $2 \mathrm{~nm}$ were 0.0268 for $30 \mathrm{~dB}, 0.0481$ for $20 \mathrm{~dB}$, and 0.0643 for $10 \mathrm{~dB}$, respectively. Similar performances were obtained from the other three kinds of dual-peak spectra. We could find that the fabricated MTF filter array performs well to reconstruct dual-peak spectra in noisy environments. 

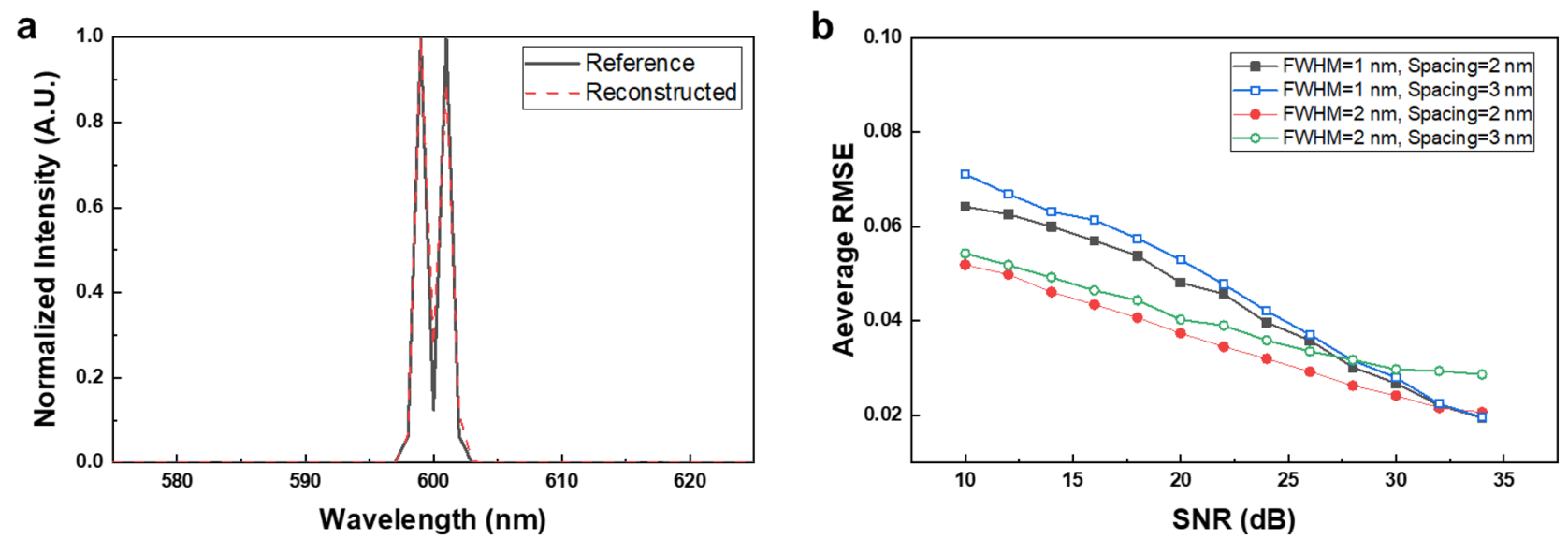

Figure 3. Simulation result of dual-peak spectra reconstructions using MTF filter array. (a) An example of dual-peak spectra with an FWHM of $1 \mathrm{~nm}$ with $2 \mathrm{~nm}$ apart. (b) Reconstruction performances of dual-peak spectra with respect to signal-to-noise ratios.

a

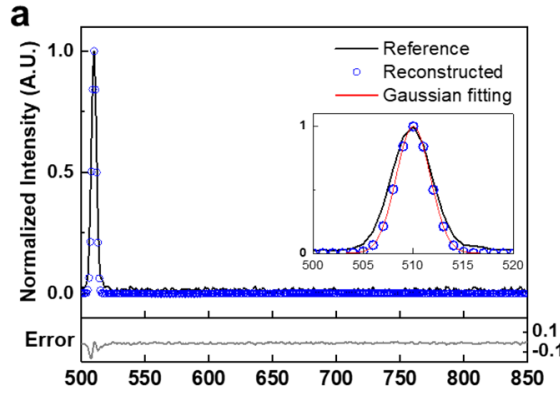

d

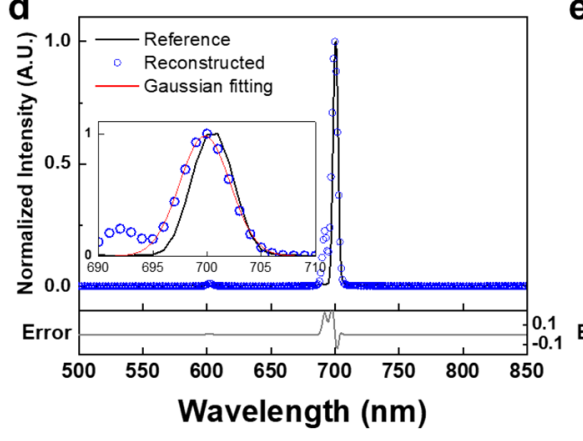

b

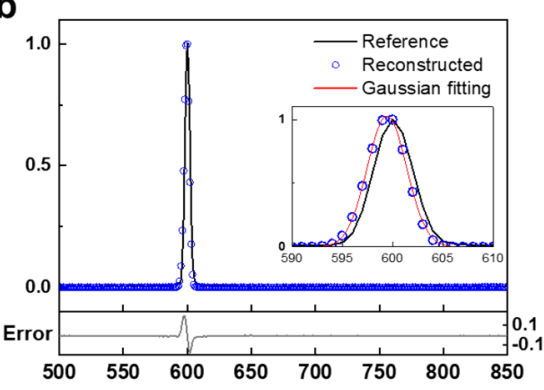

e

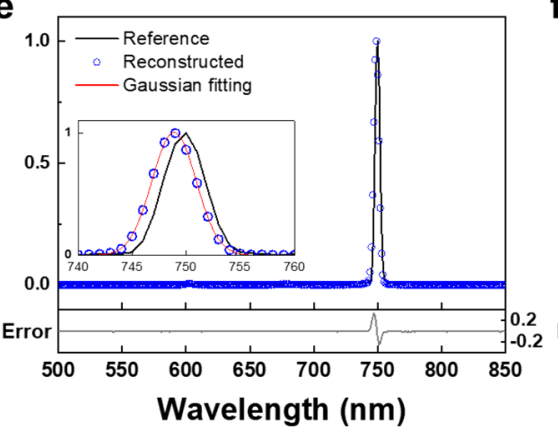

C

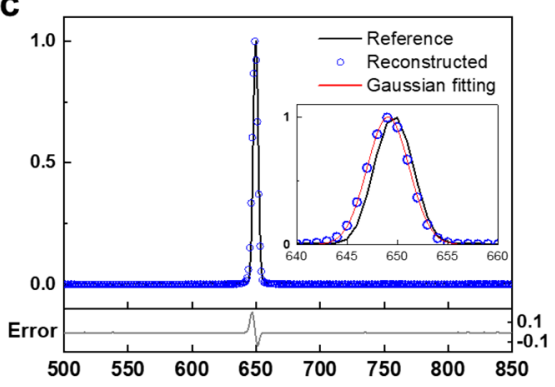

f

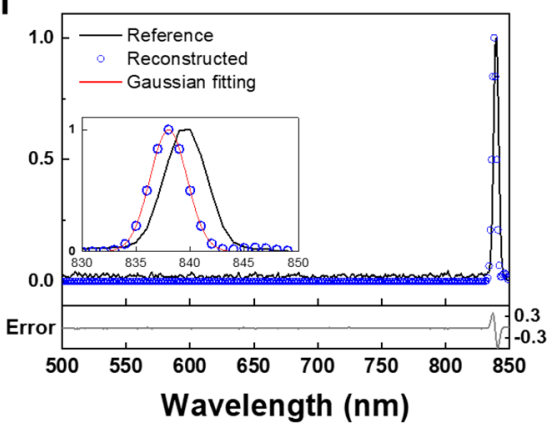

Figure 4. Spectral reconstruction of monochromatic light sources. Monochromatic light with an FWHM of $4 \mathrm{~nm}$ at peak wavelengths of (a) $510 \mathrm{~nm}$, (b) $600 \mathrm{~nm}$, (c) $650 \mathrm{~nm}$, (d) $700 \mathrm{~nm}$, (e) $750 \mathrm{~nm}$, and (f) $840 \mathrm{~nm}$. Solid black lines represent reference spectra which are measured by the grating-based spectrometer. Blue circles represent reconstructed spectra using the MTF filter array spectrometer. Solid red lines represent the results of Gaussian fitting. Solid light gray lines represent error between reconstructed and reference spectra.

After analyzing the dual-peak resolution of the MTF filter array in simulations, we tested monochromatic lights by varying the peak wavelength. The CMOS monochrome camera was used for measuring the intensities of test lights. The pixel size of the CMOS image sensor is $5.2 \times 5.2 \mu \mathrm{m}$. Underneath each filter, there are approximately $60 \times 60$ pixels. However, considering a case where the layer's location mismatch may occur during the fabrication process of the MTF filter, we excluded the boundary pixels. The averaged intensities from $30 \times 30$ pixels at the center of each filter were used for the spectral reconstruction experiments. Using a grating-based spectrometer (Black-Comet, StellarNet), monochromatic lights were measured for use as a reference.

Figure 4 shows the reconstruction results for the monochromatic light. For ease of comparison, reference spectra and reconstructed spectra are normalized. Solid black lines and blue circles in Fig. 4 represent reference spectra and reconstructed spectra, respectively. Reference spectra have peak wavelengths at 510, 600, 650, 700,750 and $840 \mathrm{~nm}$ with FWHMs of $4 \mathrm{~nm}$. As depicted in the inset enlarged graph, the reconstructed spectra using the MTF filter array spectrometer matched the reference spectra. More specifically, differences of peak wavelengths between reference and reconstructed spectra were within $2 \mathrm{~nm}$. The RMSEs were 0.023, 0.023, 


\begin{tabular}{|l|r|r|r|r|r|r|}
\hline Monochromatic light & $\mathbf{5 1 0} \mathbf{n m}$ & $\mathbf{6 0 0} \mathbf{n m}$ & $\mathbf{6 5 0} \mathbf{n m}$ & $\mathbf{7 0 0} \mathbf{n m}$ & $\mathbf{7 5 0} \mathbf{n m}$ & $\mathbf{8 4 0} \mathbf{n m}$ \\
\hline Peak Center (nm) & 509.995 & 599.438 & 649.092 & 699.808 & 748.878 & 838.001 \\
\hline Peak shift (nm) & 0.005 & 0.562 & 0.908 & 0.192 & 1.122 & 1.999 \\
\hline FWHM (nm) & 4.008 & 4.597 & 4.887 & 5.486 & 4.79 & 4.003 \\
\hline
\end{tabular}

Table 1. Evaluation of monochromatic lights reconstructions using the Gaussian curve fitting.
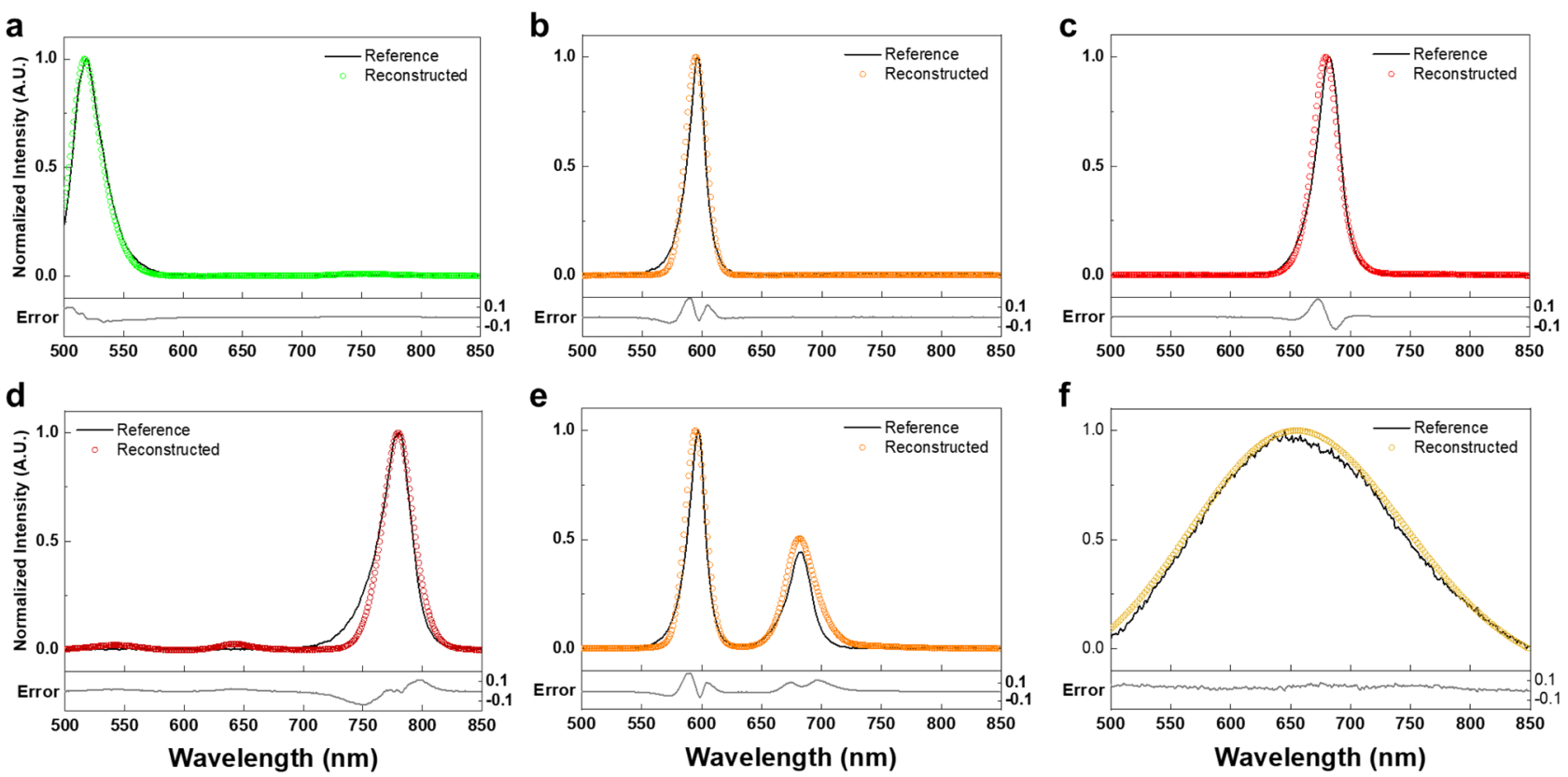

Figure 5. Spectral reconstructions of LEDs and a halogen light source. (a) a green LED, (b) an orange LED, (c) a red LED, (d) an infrared LED, (e) Combined two LEDs (orange and red), and (f) a halogen light source. Solid black lines represent reference spectra which are measured by the grating-based spectrometer. Colored circles represent reconstructed spectra using the MTF filter array spectrometer. Solid light gray lines represent error between reconstructed and reference spectra.

$0.021,0.035,0.035$, and 0.061 , for wavelengths $510,600,650,700,750$, and $840 \mathrm{~nm}$, respectively, as shown in Fig. $4 \mathrm{a}-\mathrm{f}$. Table 1 presents the evaluation of monochromatic light reconstructions using Gaussian fittings. Over monochromatic lights, peak shifts and FWHMs were within $2 \mathrm{~nm}$ and $5.5 \mathrm{~nm}$, respectively. Spectral reconstruction performance seems to degrade in the long-wavelength range due to the low spectral response of the CMOS image sensor and the monotonous spectral features of MTF filters.

We further explored the performance of the MTF filter array spectrometer using broadband light sources, such as LEDs and a halogen light source. Figure 5 shows the spectral reconstruction results. Solid black lines represent reference spectra, which are measured using the grating-based spectrometer. Colored circles represent reconstructed spectra using the MTF filter array spectrometer. Three single-color visible LEDs and one singlecolor infrared LED were used for spectral reconstruction experiments, as shown in Fig. 5a-d. A green LED (LED 525E, Thorlabs) with an FWHM of $32 \mathrm{~nm}$ was reconstructed with an RMSE of 0.021. An orange LED (LED 600L, Thorlabs) with an FWHM of $12 \mathrm{~nm}$ was reconstructed with an RMSE of 0.034. A red LED (LED 680L, Thorlabs) with an FWHM of $16 \mathrm{~nm}$ was reconstructed with an RMSE of 0.035. An infrared LED (LED 780E, Thorlabs) with an FWHM of $25 \mathrm{~nm}$ was reconstructed with an RMSE of 0.044 . Similar to experimental results of monochromatic light, the reconstruction performance is relatively poor for spectrum in the long-wavelength range. In addition, we conducted the spectral reconstruction for combined LEDs (an orange LED and a red LED), as shown in Fig. 5e. A beam splitter is used to measure the light of the combined LED. The combined LED was reconstructed with an RMSE of 0.044 . Finally, the spectral reconstruction of the halogen light source was conducted. The halogen light source, with an FWHM of $180 \mathrm{~nm}$, was reconstructed with an RMSE of 0.034 , as shown in Fig. 5f. As evidenced by low RMSE values, reconstructed spectra agree well with reference spectra measured by the grating-based spectrometer.

Computational pinhole spectral imaging. Furthermore, we demonstrated spectral imaging using the MTF filter array. As shown in Fig. 6a, the pinhole imaging system was constructed by combining a pinhole (Edmund optics), whose aperture diameter is $150 \mu \mathrm{m}$, with the monochrome CMOS image camera. The MTF filter array was placed in front of the pinhole. A single filter was adjusted to the pinhole to allow an incident image to pass through the filter and pinhole, and the filtered image was measured by the CMOS image sensor. 
a

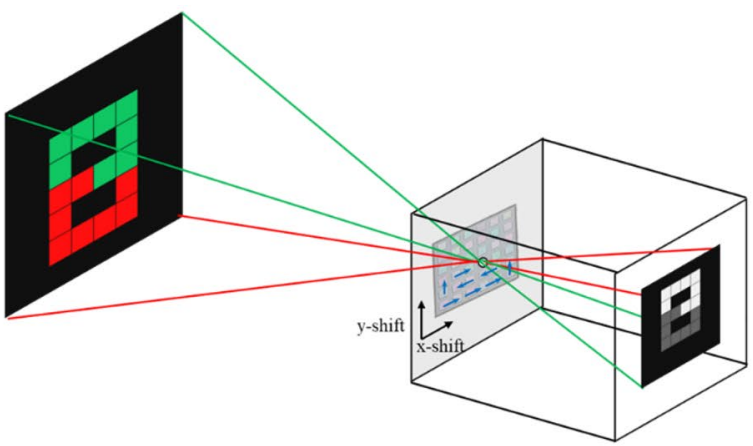

b

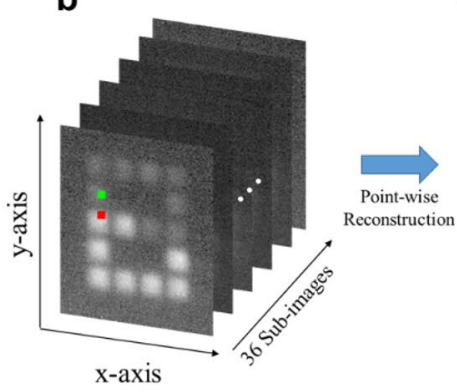

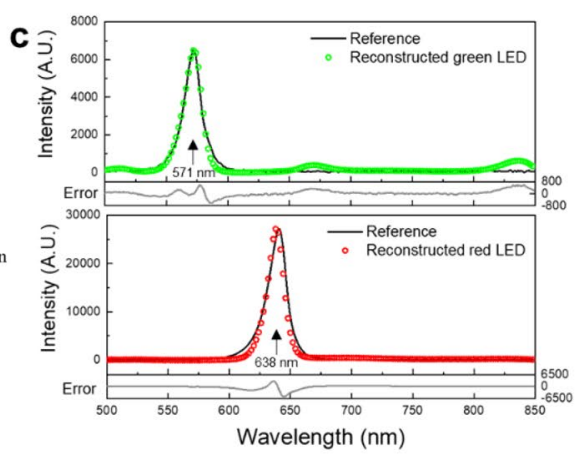

d

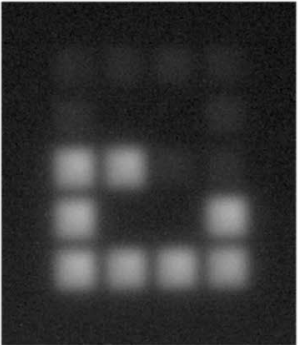

Reference

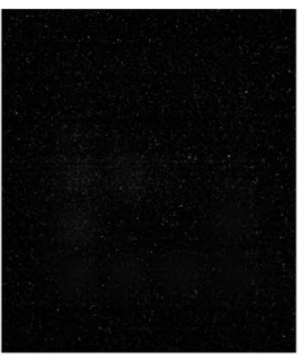

$600 \mathrm{~nm}$

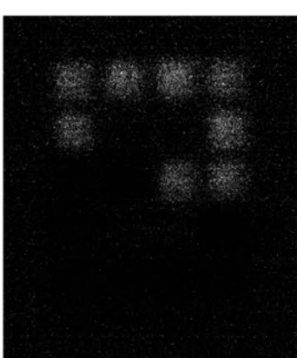

$571 \mathrm{~nm}$

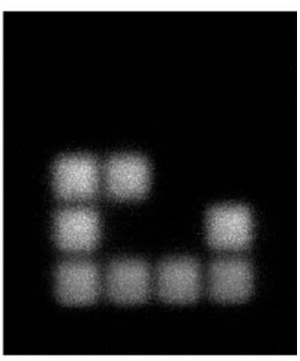

$638 \mathrm{~nm}$

Figure 6. Computational pinhole spectral imaging. (a) Schematic of pinhole imaging; The MTF filter array is placed in front of the pinhole camera. A single filter is adjusted to the pinhole and the filtered image is acquired. By changing filters, 36 filtered images are obtained. (b) 36 filtered images of $8 \times 8$ LED matrix showing the number " 8 ". The upper part consists of green LEDs and the lower part consists of red LEDs. (c) Point-wise spectral reconstruction: a pixel of a green LED block and a red LED block which are denoted in (b). Solid light gray lines represent error between reconstructed and reference. (d) Monochrome image of reference and reconstructed monochrome images at 571, 600 and $638 \mathrm{~nm}$, respectively.

By changing filters using a linear translation stage (Newport), 36 filtered images are obtained. Bi-Color $8 \times 8 \mathrm{LED}$ matrix (Adafruit) was used to generate a target. We made a small display by connecting the LED matrix to an Arduino Uno (Arduino) and by controlling the color of the 64 blocks. The number "8" was represented by the LED cube. The upper blocks consist of green LEDs, and the lower blocks consist of red LEDs. Figure $6 \mathrm{~b}$ shows a stack of the filtered 36 sub-images. A $1280 \times 1024$ size image was reduced to a sub-image size of $350 \times 300$ by discarding unnecessary pixels. Thus, a data cube with $350 \times 300 \times 36$ in size was obtained. Spectral reconstruction was performed for each pixel, and the data cube was restored with a size of $350 \times 300 \times 350$. It took $\sim 1.8 \mathrm{~h}$ to reconstruct the data cube. As shown in Fig. 6c, the reference spectra measured by the grating-based spectrometer are shown in solid black lines. As denoted pixels in Fig. 6b, the reconstructed spectra of a pixel in the green LED block and a pixel in the red LED block are represented in Fig. $6 \mathrm{c}$ as green circles and red circles, respectively. The RMSE was calculated after normalizing reference spectra and reconstructed spectra. The green LED with an FWHM of $15 \mathrm{~nm}$ was reconstructed with an RMSE of 0.0315 . The red LED with an FWHM of $20 \mathrm{~nm}$ was reconstructed with an RMSE of 0.0370 . Figure $6 \mathrm{~d}$ shows the monochrome image of reference and reconstructed monochrome images at 571,600 , and $638 \mathrm{~nm}$. The pinhole imaging system also measured the monochrome image of reference without the MTF filter array. Since the spectral component of the red LED does not exist at $571 \mathrm{~nm}$, only the upper blocks of the number " 8 " are shown in the reconstructed monochrome image at $571 \mathrm{~nm}$. On the other hand, only the lower blocks of the number " 8 " are shown in the reconstructed monochrome image at $638 \mathrm{~nm}$, where the spectral component of the green LED does not exist. Finally, nothing is displayed in the reconstructed monochrome image at $600 \mathrm{~nm}$, where spectral components of the green and red LED do not exist.

As proof-of-principle of the spectral imaging, we implemented the spectral scanning method on the pinhole imaging system. While reconstructed spectra of the pinhole spectral imaging match well with reference spectra, there are improvements to consider. In the spectral scanning method, the data cube acquisition time is long so that spectral smearing may occur in the case of a moving target. The non-scanning method, such as in snapshot spectral imaging systems, can solve these problems by acquiring the data cube in a single exposure. We assume that it is possible to construct a snapshot spectral imaging system combining the MTF filter array and a thin observation module by bound optics (TOMBO $)^{32-34}$ structure. This spectral imaging system requires a microlens array and a single separator but does not need the MTF filter to be as small as pixel size. Rather, the MTF filter should be made large so that many pixels are underlying the filter. The MTF filter array can be fabricated in scalable using stencil lithography techniques according to the spatial resolution of the spectral imaging system. 


\section{Discussion}

In conclusion, we mass-produced MTF filter arrays using stencil lithography and experimentally demonstrated the spectral resolvability of an MTF filter array-based computational spectrometer. 169 identical filter arrays with 36 MTF filters were fabricated on a single wafer. Although the MTF filter size was larger than that of the photonic crystal slabs ${ }^{14,16}$, it can be improved to a smaller size by using advanced lithography techniques and facilities. In addition, by using a higher refractive index material, the number of layers of the MTF filter can be reduced so that manufacturing efficiency can be improved.

Using the random spectral features of MTF filters and numerical optimization techniques, we recover varied spectra from the visible range to the near-infrared range (500 to $849 \mathrm{~nm}$ ) with $1 \mathrm{~nm}$ spacing. The spectral reconstruction performance in the near-infrared range is relatively inferior to the visible range, but it can be further improved by using a CMOS image sensor with a high spectral response in the near-infrared region. Also, computational spectral imaging with the MTF filter array was demonstrated using the spectral scanning method. The reconstructed data cube was found to match well with spatial and spectral references. However, to use the spectral imaging system in mobile applications, a shorter data cube acquisition time is required. By utilizing the TOMBO structure with the MTF filter array, it is possible to construct a snapshot spectral imaging system that has a short acquisition time.

Finally, the production of the MTF filter arrays is an important step towards the industrialization and practical uses of computational spectrometers. This study will be helpful for computational spectroscopy to be used in various applications where compact size, high resolution, and wide working range are required.

\section{Methods}

Simulation details. Using Gaussian distribution functions, we generated a dual-peak spectrum $\mathbf{x}$ as shown in Fig. 3a. The spectrum $\mathbf{x}$ is computationally measured as $\mathbf{y}$ by multiplying the sensing matrix $\mathbf{R}$, i.e., $\mathbf{y}=\mathbf{R x}$. In addition, we made noisy measurement $\tilde{\mathbf{y}}$ by adding additive noise $\mathbf{n}$ as $\tilde{\mathbf{y}}=\mathbf{y}+\mathbf{n}=\mathbf{R x}+\mathbf{n}$. SNR in decibels is defined as $10 \log \left(\|\mathbf{x}\|_{2}^{2} / N \sigma^{2}\right)$ where $\sigma$ is the standard deviation of the noise.

\section{Data availability}

Raw data is available from the corresponding author upon reasonable request.

Received: 6 September 2021; Accepted: 8 February 2022

Published online: 08 March 2022

\section{References}

1. Clark, R. N. \& Roush, T. L. Reflectance spectroscopy: Quantitative analysis techniques for remote sensing applications. J. Geophys. Res. Solid Earth 89, 6329-6340 (1984).

2. Bacon, C. P., Mattley, Y. \& DeFrece, R. Miniature spectroscopic instrumentation: Applications to biology and chemistry. Rev. Sci. Instrum. 75, 1-16 (2004).

3. Kim, S. et al. Smartphone-based multispectral imaging: System development and potential for mobile skin diagnosis. Biomed. Opt. Express 7, 5294-5307 (2016).

4. Kurokawa, U., Choi, B. I. \& Chang, C.-C. Filter-based miniature spectrometers: Spectrum reconstruction using adaptive regularization. IEEE Sens. J. 11, 1556-1563 (2011).

5. Oliver, J., Lee, W., Park, S. \& Lee, H.-N. Improving resolution of miniature spectrometers by exploiting sparse nature of signals. Opt. Express 20, 2613-2625 (2012).

6. Oliver, J., Lee, W.-B. \& Lee, H.-N. Filters with random transmittance for improving resolution in filter-array-based spectrometers. Opt. Express 21, 3969-3989 (2013).

7. Wang, Z. \& Yu, Z. Spectral analysis based on compressive sensing in nanophotonic structures. Opt. Express 22, 25608-25614 (2014).

8. Bao, J. \& Bawendi, M. G. A colloidal quantum dot spectrometer. Nature 523, 67-70 (2015).

9. Yang, T. et al. Miniature spectrometer based on diffraction in a dispersive hole array. Opt. Lett. 40, 3217-3220 (2015).

10. Huang, E., Ma, Q. \& Liu, Z. Etalon array reconstructive spectrometry. Sci. Rep. https://doi.org/10.1038/srep40693 (2017).

11. Oiknine, Y., August, I., Blumberg, D. G. \& Stern, A. Compressive sensing resonator spectroscopy. Opt. Lett. 42, 25-28 (2017).

12. Cerjan, B. \& Halas, N. J. Toward a nanophotonic nose: A compressive sensing-enhanced, optoelectronic mid-infrared Spectrometer. ACS Photonics 6, 79-86 (2018).

13. Kim, C., Lee, W.-B., Lee, S. K., Lee, Y. T. \& Lee, H.-N. Fabrication of 2D thin-film filter-array for compressive sensing spectroscopy. Opt. Lasers Eng. 115, 53-58 (2019).

14. Wang, Z. et al. Single-shot on-chip spectral sensors based on photonic crystal slabs. Nat. Commun. 10, 1020 (2019).

15. Yang, Z. et al. Single-nanowire spectrometers. Science 365, 1017-1020 (2019).

16. Zhu, Y., Lei, X., Wang, K. X. \& Yu, Z. Compact CMOS spectral sensor for the visible spectrum. Photonics Res. 7, 961-966 (2019).

17. Yang, Z., Albrow-Owen, T., Cai, W. \& Hasan, T. Miniaturization of optical spectrometers. Science 371, 0722 (2021).

18. August, Y. \& Stern, A. Compressive sensing spectrometry based on liquid crystal devices. Opt. Lett. 38, 4996-4999 (2013).

19. Kwak, Y., Park, S. M., Ku, Z., Urbas, A. \& Kim, Y. L. A pearl spectrometer. Nano Lett. 21, 921 (2020).

20. Liu, C. \& Sun, Z. Design and fabrication of a metallic irregular F-P filter array for a miniature spectrometer. Appl. Opt. 60, 4948-4953 (2021).

21. Li, H. et al. A near-infrared miniature quantum dot spectrometer. Adv. Opt. Mater. 9, 2100376 (2021).

22. Baraniuk, R. G. Compressive sensing [lecture notes]. IEEE Signal Process. Mag. 24, 118-121 (2007).

23. Donoho, D. L. Compressed sensing. IEEE Trans. Inf. Theory 52, 1289-1306 (2006).

24. Barry, J. R. \& Kahn, J. M. Link design for nondirected wireless infrared communications. Appl. Opt. 34, 3764-3776 (1995).

25. Macleod, H. A. Thin-Film Optical Filters (CRC Press, 2001).

26. Koh, K., Kim, S.-J. \& Boyd, S. An interior-point method for large-scale 11-regularized logistic regression. J. Mach. Learn. Res. 8, 1519-1555 (2007).

27. Bioucas-Dias, J. M. \& Figueiredo, M. A. A new TwIST: Two-step iterative shrinkage/thresholding algorithms for image restoration. IEEE Trans. Image Process. 16, 2992-3004 (2007).

28. Wright, S. J., Nowak, R. D. \& Figueiredo, M. A. Sparse reconstruction by separable approximation. IEEE Trans. Signal Process. 57, 2479-2493 (2009) 
29. Wang, S.-W. et al. 128 channels of integrated filter array rapidly fabricated by using the combinatorial deposition technique. Appl. Phys. B 88, 281-284 (2007).

30. Candes, E. \& Romberg, J. l1-magic: Recovery of Sparse Signals Via Convex Programming, Vol. 4, 14 (2005) www.acm.caltech.edu/ 11 magic/downloads/limagic.pdf.

31. Koh, K., Kim, S. \& Boyd, S. l1 ls: A Matlab Solver for Large-Scale l1-Regularized Least Squares Problems (2008).

32. Tanida, J. et al. Thin observation module by bound optics (TOMBO): Concept and experimental verification. Appl. Opt. 40, 1806-1813 (2001).

33. Gupta, N., Ashe, P. R. \& Tan, S. Miniature snapshot multispectral imager. Opt. Eng. 50, 033203 (2011).

34. Geelen, B., Tack, N. \& Lambrechts, A. A snapshot multispectral imager with integrated tiled filters and optical duplication. In Advanced Fabrication Technologies for Micro/Nano Optics and Photonics VI, Vol. 8613, 861314 (International Society for Optics and Photonics, 2013).

\section{Acknowledgements}

This work was supported by a National Research Foundation of Korea (NRF) Grant funded by the Korean government (MSIP) (NRF-2021R1A2B5B03002118).

\section{Author contributions}

C.K. and H.-N.L. conceptualized the idea. C.K., P.N. and K.R.L. performed the design and fabrication of the MTF filter arrays. C.K. and P.N. conducted optical experiments and reconstructions. C.K. visualized the experiments results. All authors wrote the manuscript.

\section{Competing interests}

The authors declare no competing interests.

\section{Additional information}

Supplementary Information The online version contains supplementary material available at https://doi.org/ 10.1038/s41598-022-08037-y.

Correspondence and requests for materials should be addressed to H.-N.L.

Reprints and permissions information is available at www.nature.com/reprints.

Publisher's note Springer Nature remains neutral with regard to jurisdictional claims in published maps and institutional affiliations.

(c) (i) Open Access This article is licensed under a Creative Commons Attribution 4.0 International License, which permits use, sharing, adaptation, distribution and reproduction in any medium or format, as long as you give appropriate credit to the original author(s) and the source, provide a link to the Creative Commons licence, and indicate if changes were made. The images or other third party material in this article are included in the article's Creative Commons licence, unless indicated otherwise in a credit line to the material. If material is not included in the article's Creative Commons licence and your intended use is not permitted by statutory regulation or exceeds the permitted use, you will need to obtain permission directly from the copyright holder. To view a copy of this licence, visit http://creativecommons.org/licenses/by/4.0/.

(C) The Author(s) 2022 